\title{
Effect of Changes in the Breathing Mode on Dental Management of Pediatric Patient
}

\author{
Luciana de Barros Correia Fontes* \\ Department of Clinic and Preventive Dentistry, Brazil \\ *Corresponding author: Luciana de Barros Correia Fontes, Department of Clinic and Preventive Dentistry, Brazil
}

\section{Opinion}

We live the reality of global climate change, with increasing amount of environmental pollutants and worsening lung and respiratory diseases. In this context, children and the elderly represent groups with greater risk and vulnerability of negative impact on quality of life. There is still no an efficient control of this population. Considering, more specifically, the dental care for children patients, there are risk factors that may influence the management and the response of this target population, who present changes in breathing pattern. According to the scientific literature, oral or mouth breathing can be related to functional, structural, occlusal and postural impairments. The oral breathers have increased craniocervical angle, reduction of cervical lordosis, increased head elevation and greater extension of this in relation to the cervical spine. Postural alteration in the cephalic and lower limbs position with disorganization of the muscular chains and in some movements, thoracic deformities, flaccid and distended abdominal musculature, sleep disorders, chronic fatigue, daytime sleepiness and lack of concentration, reduction of muscle strength, nutritional changes and impairment of some sensory systems, with a typical visual, tactile, auditory, olfactory and gustatory processing.

All this has been reported, not to mention the characteristics related to craniofacial growth patterns, facial types, malocclusion, the presence of temporomandibular disorders, gingivitis and dental caries susceptibility, oral mucosal lesions predispose and deleterious oral habits. In the oral cavity there is a reduction of the sensorial perception, with alterations mainly related to the tonus, posture and mobility of the tongue and lips, besides other structures, which would cause an incoordination of the oral functions and the phono articulatory organs. Reflecting on what has been described previously, we question the approach of these individuals, regarding the communication and behavior

expected during dental care, particularly anxiety, the time of care and the positioning of the patient in the dental chair. In addition, remember that these patients are more susceptible to mucus vomit and may present, by age, anterior vomiting reflex. It is proposed the suggestion strategies for the pediatric dental patients: oral desensitization, reduction of possible manifestations of oral defensiveness; the dentistry procedures could be performed after nasal massage and cleansing if it is necessary and confirm the anatomical and physiological capacity of nasal breathing. It follows the reinforcement of a multidisciplinary practice of accompaniment to these patients and of further investigations on the subject. 
CC (P) This work is licensed under Creative Commons Attribution 4.0 License

To Submit Your Article Click Here: Submit Article

DOI: $10.32474 /$ IPDOAJ.2018.02.000137

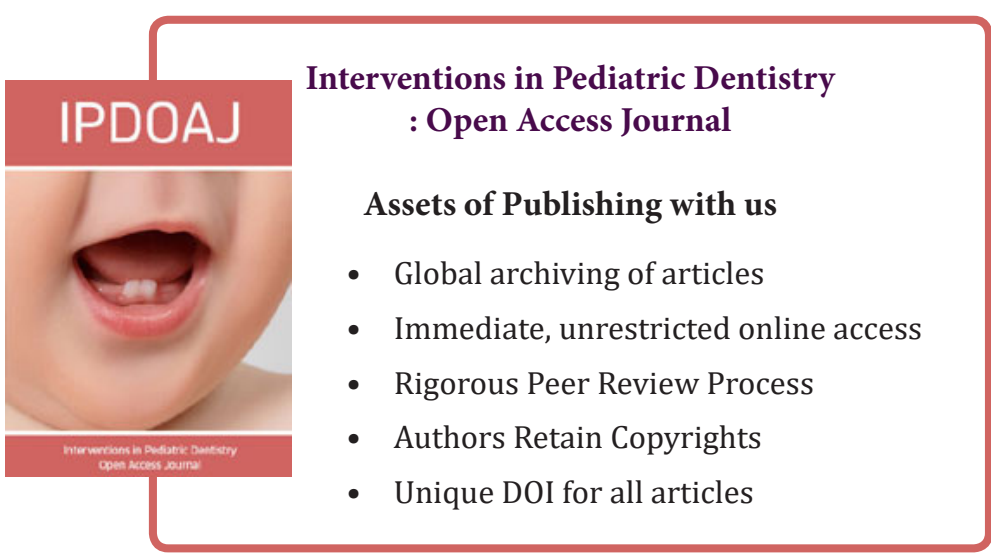

